\title{
New Generation Cooperative and Cognitive Dual Satellite Systems: Performance Evaluation
}

\author{
Shree Krishna Sharma, Dimitrios Christopoulos, Symeon Chatzinotas, and Björn Ottersten \\ Interdisciplinary Centre for Security, Reliability and Trust (SnT), University of Luxembourg \\ Email: \{shree.sharma, dimitrios.christopoulos, symeon.chatzinotas, bjorn.ottersten\}@uni.lu.
}

\begin{abstract}
Investigating innovative satellite architectures with enhanced system throughput is one of the most important challenges towards realizing the next generation of satellite communication systems. In this context, we study two advanced architectures, namely cooperative and cognitive satellite systems. These designs allow the spectral coexistence of two multibeam satellites over a common coverage area with the overlapping beam patterns. In the cooperative dual satellite system, we consider coordination between two coexisting transmitters in order to reduce the intersatellite interference. This is achieved by employing adequate user scheduling, based on the channel state information of each user. To this end, a semi-orthogonal interference aware scheduling algorithm is applied. Further, in the cognitive dual satellite system, we employ a cognitive beamhopping technique assuming that the secondary gateway is aware of the primary's beamhopping pattern. Moreover, we compare the performances of these schemes with those of the conventional multibeam and overlapping dual satellite systems in terms of spectral efficiency, power efficiency and user fairness. Finally, we provide several insights on the performance of these schemes and provide interesting future works in these domains.
\end{abstract}

Index Terms: Dual Satellite System, Cooperative Systems, Cognitive Radio Communication, Next Generation SatComs

\section{Introduction}

\section{I.A. Background}

The continuously increasing demand for multimedia, broadcast, and broadband interactive services combined with the current static frequency allocation schemes, are rendering the usable satellite spectrum scarce. Furthermore, there is continued pressure on satellite bands, especially in L and $\mathrm{C}$ bands due to the introduction of new terrestrial services such as LTE, 3G mobile telephony, WiMax and WiFi services. Due to the limited availability of L and S-band frequency resources, current research is examining the use of higher frequency bands i.e., $\mathrm{Ku}$, $\mathrm{Ka}$ bands [1] and even $\mathrm{Q} / \mathrm{V}$ bands for the feeder links. Moreover, in order to be competitive with the terrestrial networks in terms of peak data rates and capacity unit costs as well as to cope with the technical and economic demands of the broadband market, next generation Satellite Communications (SatComs) is targeting to realize the Terrabit/s satellite within the 2020 horizon [2]. In this context, it's an important challenge to investigate innovative advanced satellite architectures which can achieve enhanced system throughput by the means of higher spectral efficiency.

Towards exploiting the spatial separation of users and enhance the system throughput, satellite systems have moved from payloads generating a single beam to the multi-beam platform in which a satellite is equipped with multibeam antennas to cover multiple spots over the surface of the Earth. This multibeam architecture allows for a significant enhancement in the system capacity 
by reusing the available spectrum within the coverage area following similar to terrestrial cellular systems' principles. In a multibeam satellite system, by allowing aggressive frequency reuse over a coverage area, the available bandwidth per beam can be increased, at the expense of the increased co-channel interference. Since the system capacity depends on both the available bandwidth and the co-channel interference, enhancing the spectral efficiency of the available bandwidth and exploring advanced interference mitigation techniques in order to mitigate inter/intra-beam interferences are two main challenges towards realizing the next generation Terrabit/s SatComs. In this direction, cooperative and cognitive satellite systems can be considered as innovative and promising satellite architectures which can address the aforementioned challenges. In cooperative satellite networks, multibeam joint processing can be applied at the gateway station in order to process the spatially distributed users jointly. Consequently, multiuser encoding and decoding techniques can be utilized in order to maximize the spectral and energy efficiency $[3,4]$. In cognitive dual satellite systems, different cognitive techniques such as interweave, underlay and overlay techniques can be applied in order to allow the coexistence of two satellite systems over the same spectrum, hence enhancing the overall spectral efficiency of the satellite system [5].

\section{I.B. Motivation}

Recently, the research towards the dual satellite coexistence using cooperative and cognitive techniques is receiving important attention due to the following reasons [6,7]: (i) The primary and secondary satellites can be used for providing different services to same/different categories of users, hence enhancing the overall spectral efficiency of the system. As an example, the primary satellite with larger beams can be used to provide high priority broadband multimedia services and the secondary satellite having smaller beams to provide services requiring low Quality of Service (QoS). (ii) The Geostationary (GEO) orbit is being congested due to a significant increase in the number of GEO satellites. In this context, the cooperation between multibeam satellites can be utilized in order to overcome the issue of adjacent satellite interference. (iii) There may arise a situation where the operator has to launch another satellite that supports the existing ones in the same orbital slot in order to meet the increased traffic demand in the same coverage area. (iv) During the replacement phase of the legacy multibeam satellites, long periods of satellite coexistence are most likely to appear. This "by default" co-existence of two satellites can be exploited in order to enhance the total system capacity. Due to the aforementioned substantiation of the dual satellite coexistence, in this contribution, we are interested in analyzing the performance of cooperative and cognitive dual satellite systems operating over the same coverage area. The main difference between the cooperative and cognitive approaches is that in the cooperative approach, we exploit the benefits of full frequency reuse by applying advanced interference mitigation and user selection techniques considering partial cooperation between two satellites whilst in the cognitive approach, we exploit the spectral gaps in the primary system in order to allow the operation of the secondary system in the same spectrum without providing harmful interference to the primary system.

\section{I.C. Contributions}

In this work, we evaluate the performances of two promising technologies i.e., cooperative and cognitive techniques for the next generation SatCom systems and compare their performances with the traditional multibeam and overlapping dual multibeam satellite systems. The following performance metrics are used for comparing these schemes: (i) Spectral Efficiency (SE) in bits/s/Hz, (ii) Power Efficiency (PE) in bits/Hz/J, and (iii) user fairness in terms of Jain's fairness index [8]. In the context of cooperative techniques, a heuristic, iterative and low complexity algorithm that

allocates users in each of the two interfering sets, originally proposed in [6], is employed. In this context, the performance of each satellite can be enhanced by maximizing the orthogonality between users allocated in the same set, hence optimizing the zero forcing performance while the performance of the overall system can be improved by minimizing the level of interferences between two sets of users. In the context of cognitive satellite systems, we apply the cognitive beamhopping technique, originally proposed in [7], for the considered dual satellite spectral coexistence scenario. With the help of prior knowledge of the primary beamhopping pattern and proper synchronization, 
the secondary satellite beamhopping pattern can be designed to ensure sufficient protection to the primary system. Furthermore, we apply a power control technique as in [7] for secondary satellite transmission in order to respect the interference constraint of the primary system.

\section{I.D. Structure}

The remainder of this paper is structured as follows: Firstly, Section II presents the dual satellite channel model used in this work. Subsequently, Section III presents the cooperative dual satellite scenario and describes its system model in detail. Then Section IV provides cognitive dual satellite scenario and describes the considered cognitive beamhopping scheme in detail. The theoretical expressions for the considered schemes are included in Section V while Section VI compares the performances of the aforementioned schemes with those of the conventional multibeam and overlapping dual satellite systems. Finally, Section VII discusses future works and concludes this paper.

\section{Dual Satellite Channel Model}

In this section, we present a channel model which is common to both the considered cooperative and cognitive dual satellite systems, described later in subsequent sections. In this study, we model the satellite channel as an Additive White Gaussian Noise (AWGN) channel with real channel gains assuming the fixed satellite users equipped with highly directive antennas and clear sky conditions. The beamgain matrix, denoted by $B$, is employed for modeling the channel of each individual satellites and cross channels between two systems. The beam gain of the $j$-th beam for the $i$-th user position can be denoted by $B_{i j}$ and can be written as [9]

$$
B_{i j}=G_{\max } \cdot\left(\frac{J_{1}(u(i, j))}{2 u(i, j)}+36 \frac{J_{3}(u(i, j))^{2}}{u(i, j)^{3}}\right)^{2},
$$

where $u(i, j)=2.01723 \sin (\theta(i, j)) / \sin \left(\theta_{3 \mathrm{~dB}}\right), J_{m}$ is the first kind of Bessel's function of order $m$, and $G_{\max }$ is the maximum antenna gain, $\theta_{3 \mathrm{~dB}}$ is the 3 -dB angle and $\theta(i, j)$ represents the angular position of the $i$-th user from the $j$-th beam center with respect to the satellite. It should be noted that the maximum power is received at the beam center and the power level varies across the beam coverage area depending on the beamgain matrix given by (1). In (1), the $i$-th user and the $j$-th beam belong to the same satellite for modeling individual satellite channels whereas for the cross channels between two satellite systems, the $i$-th user belongs to the first/second satellite whereas the $j$-th beam is from the second/first satellite, respectively. Thus, by generating 4 different beam gain matrices from (1), the overall dual satellite channel is realized for the considered scenarios.

\section{Cooperative Dual Satellite System}

\section{III.A. Scenario Description}

We consider the coexistence scenario of two collocated multibeam satellites with overlapping coverage areas as shown in Fig. 1. We focus on the forward link of both satellites and consider ideal feeder links. Each satellite serves a large number of uniformly distributed users across the coverage area. For this purpose, a Time Division Multiple Access (TDMA) scheme is employed, hence one user per beam is served during each time slot. There exist two types of interferences in the considered dual-satellite system: (i) multiuser interference in each satellite, and (ii) inter-satellite interference. The multiuser interference in each satellite is handled by employing a suitable precoding technique in the transmit side [4]. As in [6], we consider Zero Forcing (ZF) beamforming with optimal in a throughput maximizing sense power allocation over the precoders. Since both multibeam satellites operate using the same set of frequencies without any frequency orthogonalization, the effect of intersatellite interference can be mitigated by employing full cooperation between their transmitters. However, the full cooperation requires a significant overhead in the capacity of the backhauling 
links between their gateways. In this context, a partial cooperation or coordination between two gateways feeding the satellites can be considered, since it reduces the amount of data to be exchanged between the gateways. This cooperation allows the coexisting transmitters to manage the inter-satellite interferences and enhance the total system performance. In this coordinated dual satellite system (Fig. 1), the satellite gateway employs multibeam joint processing. Subsequently, each user's signal is precoded at the gateway and transmitted by all on-board antennas [3]. As a result, intra-satellite interferences are mitigated. In order to manage inter-satellite interferences and thus enhance the spectral efficiency of the considered cooperative dual satellite system, a user allocation technique, originally developed in [6], is applied. Consequently, the interference between two interfering sets of users is managed.

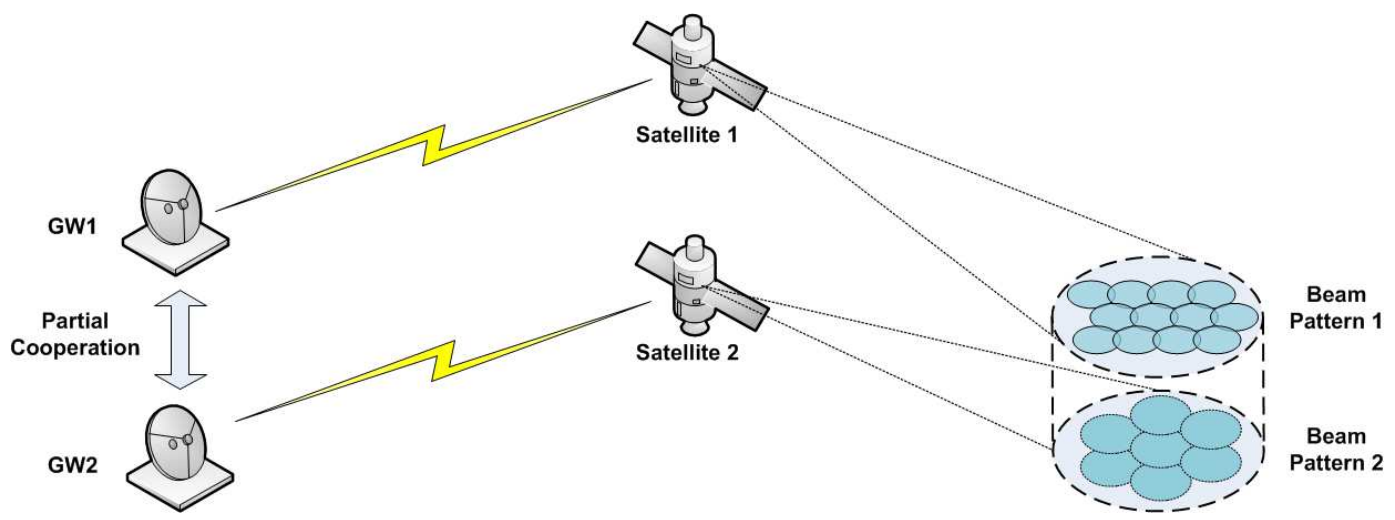

Figure 1: Dual satellite system with partial cooperation

\section{III.B. System Model}

In the existing Multiuser Multiple Input Single Output (MU-MISO) literature, precoding is considered as an interference precancelation technique which exploits the spatial degrees of freedom offered by the multiple transmit antennas $\left(N_{b}\right)$ to serve multiple single antenna users $(K)$. In the considered dual satellite multibeam system, we consider single feed per beam assumption and multiple antennas correspond to multiple feeds. The transmit signals are multiplied with precoding vectors at the transmitter in order to cancel multiuser interferences, thus creating equivalent interference free channels. For this purpose, full Channel State Information (CSI) knowledge is required at the transmitter, which may be difficult to be achieved in practice. In this context, the system performance under non-perfect CSI can be evaluated, towards a realistic system performance evaluation.

As mentioned before, we consider partial cooperation between two satellite systems and this partial cooperation only involves the exchange of the CSI instead of the full data exchange and helps to maintain the moderate system complexity. In this context, a heuristic, iterative, low complexity algorithm that allocates users in the two interfering sets is employed. This algorithm, originally proposed in [6], improves the performance of each satellite and of the overall system, simultaneously. The first performance benefit is achieved by maximizing the orthogonality between users allocated in the same set, hence optimizing the Zero Forcing (ZF) performance. On the other hand, the second performance benefit is achieved by minimizing the level of interferences between the two sets.

By considering the analogy of the MU MISO Broadcast Channel (BC), the input-output analytical expression for the $i$-th user can be written as

$$
y_{i}=\mathbf{h}_{i}^{\dagger} \mathbf{x}+z_{i}
$$

where $\mathbf{h}_{i}^{\dagger}$ is an $1 \times N_{b}$ channel vector composed of the channel coefficients (i.e., antenna gains and propagation loss) between the $i$-th user and the $N_{b}$ antennas (i.e., feeds) of the satellite, $\mathbf{x}$ is an 
$N_{b} \times 1$ transmit signal vector and $z_{i}$ is the Additive White Gaussian Noise (AWGN) measured at the $i$-th user's receive antenna.

In the considered cooperative dual satellite system, two overlapping spot-beams of $N_{b 1}$ and $N_{b 2}$ belonging to two different satellites serve $K_{1}$ and $K_{2}$ fixed user terminals located in the same coverage area. Although precoding reduces each satellite's channel into a MU-MISO broadcast channel, the dual satellite system operates over an interference channel due to intersatellite interference. A single user within the coverage area receives signal from both the satellites and hence the total channel matrix $\mathbf{H}_{\text {dual }}$ becomes of the dimensions $\left(K_{1}+K_{2}\right) \times\left(N_{b 1}+N_{b 2}\right)$. After employing precoding in each satellite to mitigate the multiuser interferences within each satellite, the SINR of the $k$-th user that belongs to the first satellite, can be written as [6]

$$
\gamma_{S 1, k}=\frac{p_{1 k}\left|\mathbf{h}_{k 1}^{\dagger} \mathbf{w}_{k 1}\right|^{2}}{1+\sum_{j=1}^{K_{2}} p_{2 j}\left|\mathbf{h}_{k 2}^{\dagger} \mathbf{w}_{j 2}\right|^{2}},
$$

where $p_{1 k}$ represents the power allocated to the $k$-th user served by the first satellite, $\mathbf{h}_{k 1}$ represents the $N_{b 1} \times 1$ channel vector of the $k$-th user towards the first satellite and $\mathbf{w}_{k 1}$ denotes the $N_{b 1} \times 1$ precoding vector for the $k$-th user served by the first satellite. Similarly, the SINR expression for the user allocated to the second satellite can be deduced by interchanging indexes in (3). Based on the above channel model, a heuristic, iterative Semi-orthogonal Interference aware User Allocation (SUIA) method proposed in [6] is applied in order to select two sets of users to be allocated to two different satellites. The basic idea behind this algorithm is to find the most orthogonal users which at the same time receive and induce the least possible interferences. It can be noted that the applied algorithm is considerably less complex and can be executed in a centralized station or run at parallel at the gateways which share the CSI.

\section{Cognitive Dual Satellite System}

\section{IV.A. Scenario Description}

Cognitive communications is considered a promising technique in order to address the satellite spectrum scarcity problem [5]. In this context, recent contributions exploit the coexistence of a satellite system with the terrestrial network $[5,11,13]$ or with another satellite network $[7,17]$. In this work, we focus on the coexistence of two collocated GEO multibeam satellite systems as shown in Fig. 2. We consider primary satellite with larger beams and secondary satellites with smaller beams operating over the same geographical region in the normal forward mode. The cognition in the considered system is achieved by sharing the beamhopping pattern and the timing information of the primary to the secondary with the help of a high speed link between their gateways.

As mentioned earlier in Section I, advanced mitigation techniques are required in order to realize the full frequency reuse due to excessive co-channel interference. Another way of realizing the full frequency reuse concept is to use a beamhopping satellite system which can operate over a certain beamhopping pattern [22]. The main difference between conventional multibeam and beamhopping systems is that frequency sharing among multiple beams within a cluster takes place in the frequency domain for the conventional multibeam system and in the time domain for the beamhopping system. Since in a beamhopping system, only a single beam of a cluster is active during a particular time slot, there exists an opportunity to reuse the full frequency using smaller beams of another secondary satellite in the same time slot. In this context, we apply the cognitive beamhooping technique proposed in [7] in the considered dual satellite coexistence scenario. The cognitive beamhopping technique allows the secondary satellite to exploit the vacant spectral opportunities by operating over the beams which are within the primary inactive beams while providing sufficient protection to the primary satellite users.

\section{IV.B. Related Literature}

Cognitive communications is considered a promising approach in order to address the spectrum scarcity problem caused by spectrum segmentation and current static frequency allocation policies. 


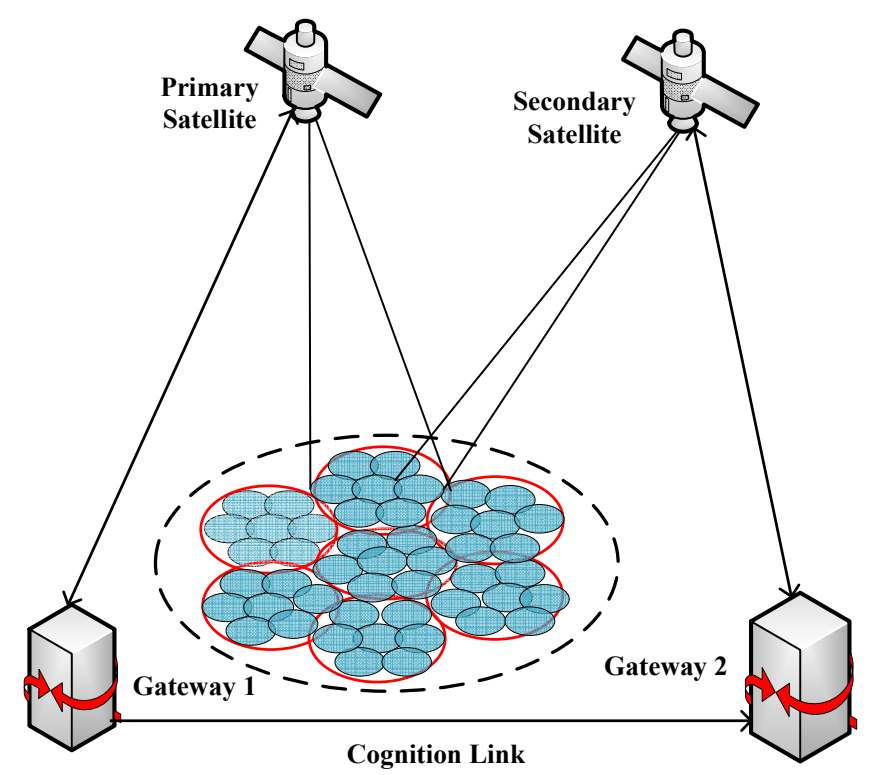

Figure 2: Dual satellite cognitive beamhopping system

Several Cognitive Radio (CR) techniques have been proposed in the literature in order to allow the coexistence of cognitive systems with the licensed primary systems. The most common cognitive techniques in the literature can be categorized into interweave or Spectrum Sensing (SS), underlay, overlay and Database (DB) related techniques [5]. The CR related research is more mature in the terrestrial context but has received limited attention in the satellite community. In this context, exploring efficient frequency sharing techniques to enhance the spectral efficiency while guaranteeing QoS in the context of SatComs is a challenging research problem. This has led to the concept of cognitive SatComs which exploits the opportunities for spectrum sharing between satellite and terrestrial systems or between two satellite systems [5]. The cognitive related satellite literature can be broadly categorized into: (i) hybrid satellite-terrestrial coexistence scenario $[5,10-13,16]$, and (ii) dual satellite coexistence scenario [7,14,15,17-20]. In [15], the polarization domain has been considered as an additional spectrum sharing dimension for dual polarized AWGN channels considering a dual satellite coexistence scenario. In [18], the problem of distributed power control has been considered for cognitive satellite networks. In [17], an interference alignment technique has been applied for the spectral coexistence of a multibeam and a monobeam satellites both operating in the normal return mode. In [20], an adaptive power control approach has been studied for allowing the coexistence of GEO and non-GEO satellites both in the uplink and downlink modes. Recently, the FP7 project Cognitive Radio for Satellite Communications "CoRaSat" is examining three different coexistence scenarios in the Ka band in order enhance the spectral efficiency of future Ka-band satellite systems [21]. In the context of beamhopping satellite systems, several contributions have exploited the benefits of this approach in various settings [24-26].

\section{IV.C. Cognitive Beamhopping System}

In a beamhopping multibeam system, a fixed number of beams are simultaneously illuminated with a regular repetition pattern. This beamhopping technique helps to reduce the number of amplifiers on board as well as the power demands on the payloads and can be implemented with full frequency or partial frequency reuse [27]. In this work, we focus on full frequency reuse as in [27].

We consider a dual satellite coexistence scenario depicted in Fig. 2. We consider both satellites to be multibeam, covering the same geographical region and operating in the normal forward mode [5]. Both satellites are assumed to be co-located in the same GEO orbit and are connected to different gateways on the Earth. These gateways are connected with the help of a high speed 
terrestrial link (e.g., optical fiber, microwave). We consider a coverage area with larger primary beams and many spot-beams within each primary beam and these spot-beams are the beams of the secondary satellite. Due to recent advances in multibeam antenna technology, it is feasible to create coverage cells of less than $0.5^{\circ}$ diameter [28], which corresponds to the approximate beam radius of $155 \mathrm{~km}$ on the surface of the Earth. The cognition is achieved by sharing the beamhopping pattern and the timing information of the primary satellite to the secondary satellite with the help of a signalling link between the gateways. Based on this a priori knowledge of the beamhopping pattern, the secondary satellite adapts its beamhopping pattern in such a way that the primary's satellite's performance is not degraded. Furthermore, the primary and secondary transmissions can be synchronized with the help of the exchanged timing information. However, symbol level synchronization is not required in this case and synchronization at the frame level during the beginning and end of the transmission is sufficient to realize a cognitive beamhopping system. The secondary satellite is supposed to be more dynamic and be equipped with smaller transponders. Further, since the higher peak gain for the spot-beams in the secondary system allows designers to have flexibility of using less transmit power per beam while meeting the beam edge requirements, designers can save payload weight on the secondary satellite in comparison to the primary satellite [27].

For the cognitive coexistence of two satellites within the same spectrum while covering the same geographical region, the footprint of these satellites should be taken into account. In this work, the channel model based on the beamgain matrix, given by (1) is followed. Since only a certain fraction of total available beams are active in a particular time slot, we can explore the possibility of using those frequencies in the secondary satellite system in a secondary way. The primary system is a beamhopping system with larger beams with slot reuse factor of $M$. The secondary system can also be considered to be a beamhopping system with smaller beams and lower transmit power. In the considered cognitive beamhopping system, the expression for the SINR of the $i$-th primary user in the presence of secondary satellite system, let us denote by $\gamma_{\mathrm{CP}, i}$, is given by

$$
\gamma_{\mathrm{cp}, i}=\frac{\left|h_{\mathrm{P}, i i}\right|^{2} P_{\mathrm{pt}}}{P_{\mathrm{pt}} \sum_{j \in S_{\mathrm{P}}}\left|h_{\mathrm{p}, i j}\right|^{2}+P_{\mathrm{st}} \sum_{j \in S_{\mathrm{S}}}\left|h_{i j, \mathrm{~s}}\right|^{2}+\sigma_{i}^{2}},
$$

where $P_{\mathrm{pt}}$ is the per beam transmit power of the primary system, $P_{\mathrm{st}}$ is the per beam transmit power of the secondary system, $S_{\mathrm{P}}$ represents the set of primary co-channel beams other than the considered beam, $S_{\mathrm{S}}$ represents the set of secondary active beams in a particular slot. The parameter $h_{\mathrm{p}, i j}$ represents the channel gain of the $i$-th PU from the $j$-th primary beam and $h_{i j, \mathrm{~s}}$ denotes the channel gain of the $i$-th PU from the $j$-th secondary beam. Similarly, the SINR of the $i$-th secondary user in the presence of primary satellite can be written as

$$
\gamma_{\mathrm{cs}, i}=\frac{\left|h_{\mathrm{s}, i i}\right|^{2} P_{\mathrm{st}}}{P_{\mathrm{pt}} \sum_{j \in S_{\mathrm{p}}}\left|h_{i j, \mathrm{p}}\right|^{2}+P_{\mathrm{st}} \sum_{j \in S_{\mathrm{S}}}\left|h_{\mathrm{s}, i j}\right|^{2}+\sigma_{i}^{2}},
$$

where $h_{i j, \mathrm{p}}$ is the channel gain of $i$-th SU from the $j$-th primary beam and $h_{\mathrm{s}, i j}$ is the channel gain of $i$-th SU from the $j$-th secondary beam.

In the considered cognitive beamhopping system, we can apply several CR techniques in order to protect the licensed primary satellite users. It should be noted that although cognitive beamhopping allows the secondary satellite to avoid active beams in the primary satellite, there may occur interference from the secondary satellite system to the licensed primary users. In this context, authors in [27] study power control and Exclusion Zone (EZ) based techniques for allowing the dual satellite coexistence employing the cognitive beamhopping technique. In the comparative study considered in this paper, we apply the power control approach as follows: Let $I_{\mathrm{T}}$ be the interference threshold level of the PU to have sufficient protection and $I_{\text {agg }}$ be the aggregate interference from secondary beams to the primary user. Then the expression for $I_{\text {agg }}$ at a particular slot can be written as: $I_{\text {agg }}=P_{\text {st }} \sum_{j \in S_{\mathrm{S}}}\left|h_{i j, \mathrm{~s}}\right|^{2}$. The transmit power of the secondary satellite can be adjusted in the following way to guarantee sufficient protection for the PU

$$
P_{\mathrm{st}}=\frac{I_{\mathrm{T}}}{\sum_{j \in S_{\mathrm{S}}}\left|h_{i j, \mathrm{~s}}\right|^{2}} \text {. }
$$


It can be noted that as the number of SUs within an primary inactive beam increases, the denominator term of the above equation increases and the secondary satellite has to reduce its transmission power.

\section{Performance Analysis}

In this section, we present throughput expressions for the considered schemes which are used for their performance assessment in Section VI.

\section{V.A. Conventional Multibeam System}

In conventional multibeam systems, the total available bandwidth $(B)$ in the forward link is divided into $M$ segments, where the parameter $M$ is the frequency reuse factor. Then the bandwidth allocated to the $i$-th user beam $\left(B_{i}\right)$ can be written as: $B_{i}=B / M$. The set of the beams which share the total bandwidth defines a beam cluster. For throughput analysis, we consider the multibeam satellite coverage with frequency reuse factor of $M$. The dominant interference in this case is the co-channel interference from neighboring co-channel cells. The SINR of the $i$-th user is given by

$$
\gamma_{\mathrm{cv}, i}=\frac{\left|h_{i i}\right|^{2} P_{\mathrm{t}}}{P_{\mathrm{t}} \sum_{j \in S_{\mathrm{P}}}\left|h_{i j}\right|^{2}+\sigma_{i}^{2}},
$$

where $P_{\mathrm{t}}$ denotes the per beam transmit power. It can be noted that we include all possible cochannel cells in a given area and adjacent channel interference is not included in (7). The system throughput for this system can be written as [23]

$$
C_{\mathrm{cv}}=\frac{B}{M} \sum_{i=1}^{N_{b}} \log _{2}\left(1+\gamma_{\mathrm{CV}, i}\right),
$$

where $N_{\mathrm{b}}$ denotes the number of beams in the considered conventional multibeam system.

\section{V.B. Overlapping Dual Multibeam System}

In this system, two collocated multibeam satellite systems operate over the same coverage region with each satellite using half of the total available spectrum. The total throughput of this system can be written as

$$
C_{\mathrm{id}}=\frac{B}{2}\left(\frac{1}{M} \sum_{i=1}^{N_{b 1}} \log _{2}\left(1+\gamma_{\mathrm{S}_{1}, i}\right)+\frac{1}{M} \sum_{i=1}^{N_{b 2}} \log _{2}\left(1+\gamma_{\mathrm{S}_{2}, i}\right)\right) .
$$

where $\gamma_{\mathrm{S}_{1}, i}$ denotes the SINR of the $i$-th user served by the first satellite and $\gamma_{\mathrm{S}_{2}, i}$ denotes the SINR of the $i$-th user served by the second satellite.

\section{V.C. Coordinated Dual Satellite System}

The total system throughput of the considered coordinated dual satellite system is given by

$$
C_{\text {cop }}=B\left(\sum_{i \in S_{\mathrm{S}_{1}}} \log _{2}\left(1+\gamma_{\mathrm{S}_{1}, i}\right)+\sum_{i \in S_{\mathrm{S}_{2}}} \log _{2}\left(1+\gamma_{\mathrm{S}_{2}, i}\right)\right),
$$

where $S_{\mathrm{S}_{1}}$ denotes the set of users allocated to the first satellite and $S_{\mathrm{S}_{2}}$ represents the set of the users allocated to the second satellite. Further, $\gamma_{\mathrm{S}_{1}, i}$ denotes the SINR of the $i$-th user allocated to the first satellite, given by (3) and $\gamma_{\mathrm{S}_{2}, i}$ denotes the SINR of the $i$-th user allocated to the second satellite, obtained by interchanging indexes in (3). 


\section{V.D. Cognitive Beamhopping System}

The total system throughput in this system can be expressed as

$$
C_{\mathrm{cb}}=C_{\mathrm{ps}}+C_{\mathrm{sp}}=B\left(\sum_{i=1}^{N_{\mathrm{b} 1} / M} \log _{2}\left(1+\gamma_{\mathrm{cp}, i}\right)+\sum_{i=1}^{N_{\mathrm{s}}} \log _{2}\left(1+\gamma_{\mathrm{cs}, i}\right)\right),
$$

where $C_{\mathrm{ps}}$ and $C_{\mathrm{sp}}$ denote primary/secondary throughput in the presence of secondary/primary system respectively, $\gamma_{\mathrm{cp}, i}$ represents the SINR of the primary user given by (4), $\gamma_{\mathrm{cs}, i}$ represents the SINR of the secondary user given by (5), $M$ denotes the slot reuse factor and $N_{s}$ denotes the number of active secondary beams.

\section{Numerical Results}

In this section, firstly, we evaluate and compare the performance of cooperative and cognitive dual satellite systems with the conventional multibeam and overlapping dual satellite systems in terms of spectral efficiency (bits/s/Hz). For this purpose, we use the same total power budget in order to make the fair comparison of different systems. Further, we compare the performance of these systems with the MU MIMO channel capacity as an upper bound. Subsequently, we provide their performance comparison in terms of power spectral efficiency (bits $/ \mathrm{Hz} / \mathrm{J}$ ) versus total power budget of the system in order to demonstrate which technique is more beneficial in terms of the power efficiency. Finally, we provide their comparison in terms of user fairness in order to illustrate which technique provides better fairness while serving randomly distributed users within the considered coverage area. There exist several ways of measuring user fairness and system fairness in wireless networks [29]. In this work, we use the term "user fairness" in order to illustrate whether the users are fairly treated by the system or not. For this purpose, we apply Jain's index as a measure for the user's rate fairness, which is defined as [8]

$$
I_{F}=\frac{\left(\sum_{i=1}^{N} x_{i}\right)^{2}}{N \sum_{i=1}^{N} x_{i}^{2}}
$$

where $N$ denotes the number of users over the considered coverage area and $x_{i}$ denotes the rate of the $i$-th user. The value of $I_{F}$ in (12) ranges from $1 / N$ (the worst case) to 1 (the best case).

In the considered simulation set up, a circular geographical region with an area radius of 3000 $\mathrm{km}$ has been considered. The considered area is divided into 7 larger beams with $3 \mathrm{~dB}$ beamwidth of $1.83^{\circ}$ and each larger beam contains 7 smaller beams with $3 \mathrm{~dB}$ beamwidth of $0.707^{\circ}$. The performance of the following four different systems have been compared in our results: (i) conventional multibeam system, (Section V.A) (ii) overlapping dual satellite system (Section V.B), (iii) cooperative dual satellite system (Section V.C), (iv) cognitive beamhopping system with and without power control (Section V.D). For simulating the conventional multibeam system, the primary only system (i.e., only the first satellite with the 7 larger beams) is considered with the frequency reuse factor of 3. Similarly, for simulating overlapping dual satellite systems, half of the spectrum is allocated to the first satellite and another half to the second satellite. Further, for simulating the cooperative multibeam satellite system, the coordinated dual satellite system as described in Section III is considered with the user selection algorithm proposed in [6]. Finally, the cognitive beamhopping system as described in Section IV is considered with the power allocation method proposed in [27]. For the considered cognitive beamhopping system, the primary system employs the slot reuse factor of 3 and the secondary satellite adjusts its beamhopping pattern based on the primary's pattern in order to avoid the primary active beams. Further, in the power control-based approach, an interference threshold of $-117 \mathrm{dBW}$ is considered in order to respect the interference tolerance threshold of the primary satellite terminals and subsequently, the maximum power between the available power per beam and the maximum allowable transmit power is chosen as the per beam transmit power of the secondary satellite. During simulation, uniformly distributed random users were generated over the considered coverage area considering one user per beam in each Monte Carlo realization, and 20 independent Monte Carlo realizations were considered for generating the final results. 


\section{VI.A. Results}

Figure 3 presents the Spectral Efficiency (SE) versus total power budget $P_{\text {tot }}$ (available onboard) comparison of the considered schemes. From the figure, it can be noted that cognitive beamhopping based dual satellite system performs better in the lower power budget regime whereas the coordinated dual satellite system performs better in higher power budget regime. Further, it can be depicted that there exists a crossing point between the performance curves of the coordinated dual satellite system and cognitive beamhopping system (without power control) at the value of $P_{\text {tot }}=22.5 \mathrm{dBW}$. In our results, we provide the performance comparison of the considered schemes over the range of $P_{\text {tot }}$ from $-5 \mathrm{dBW}$ to $50 \mathrm{dBW}$. In practice, a suitable approach can be chosen depending on the nominal operating point for the future dual satellite systems. While comparing the SE of the cognitive beamhopping scheme with and without power control, it can be observed that the SE of the power control based cognitive approach is slightly higher at the lower values of available power whereas the performance without power control becomes better at the higher values of the total available power. The MU MIMO channel capacity curve plotted in Fig. 3 depicts the theoretical upper bound for the considered system. From the result, it can be noted that at lower values of $P_{\text {tot }}$, the performance gap between the upper bound and the considered schemes is less in comparison to the performance gap in the high $P_{\text {tot }}$ region. Further, in higher $P_{\text {tot }}$ region, the performance of the coordinated approach is closer to the theoretical upper bound in comparison to other techniques.

Similarly, Figure 4 depicts the Power Efficiency (PE) versus total power comparison of the considered schemes. The PE for each scheme is obtained by dividing the spectral efficiency obtained in Fig. 3 by the total amount of the used power. From the figure, it can be noted that the PE of the considered cooperative scheme is better than other schemes at higher values of $P_{\text {tot }}$ i.e., above $P_{\text {tot }}=17 \mathrm{dBW}$ in Fig. 4 . It can be observed that the cognitive beamhopping with power control performs better than cognitive beamhopping without power control and further performs better than all other realizable schemes at the lower values of $P_{\text {tot }}$. Moreover, in terms of the PE, the performance of the overlapping dual satellite system is the worst. Finally, the performance of the conventional multibeam is better than that of the overlapping dual satellite system. When power efficiency is the goal, then the cognitive beamhopping with power control appears to be the best choice.

To compare the user fairness of the considered schemes, we plot the Cumulative Distribution Function (CDF) curves of SEs provided by these schemes at the value of $P_{\text {tot }}=22.5 \mathrm{dBW}$ in Fig. 5. This point is chosen as the crossing point for cognitive and cooperative approaches in the SE performance curve depicted in Fig. 3. In practice, a suitable operating point can be investigated for the considered dual satellite system. Further, we compute the Jain's fairness index using (12) in order to compare the user fairness of the considered schemes. The Jain's fairness index for overlapping dual satellite system, conventional multibeam system, cognitive beamhopping system, and coordinated multibeam systems was found to be $0.7659,0.9827,0.2540$, and 0.1274 respectively. From this result, it can be deduced that the conventional multibeam system is the best in terms of the user fairness i.e., treating all the users equally, and the overlapping dual satellite system is the second best. Further, the cognitive beamhopping system provides better user fairness than the coordinated dual satellite system. However, as noted from Fig. 5, the cooperative scheme can provide higher rate to certain users in comparison to other schemes due to the employed interference mitigation scheme. Therefore, if the user fairness is the goal in designing the system, the conventional satellite system is the best choice and if the design aim is to enhance the overall system throughput, cooperative scheme or cognitive scheme can be chosen based on the operating region of the employed dual satellite multibeam system.

\section{VI.B. Discussion}

It can be concluded that by implementing the proposed techniques, future satellite communication systems will benefit in terms of the system throughput and the spectral efficiency in comparison to the traditional multibeam systems. This provides insights for the GEO satellite operators, hence motivating them towards implementing advanced techniques such as cognitive and cooperative in order to boost the system capacity to achieve the target of Terrabit/s broadband capacity. 


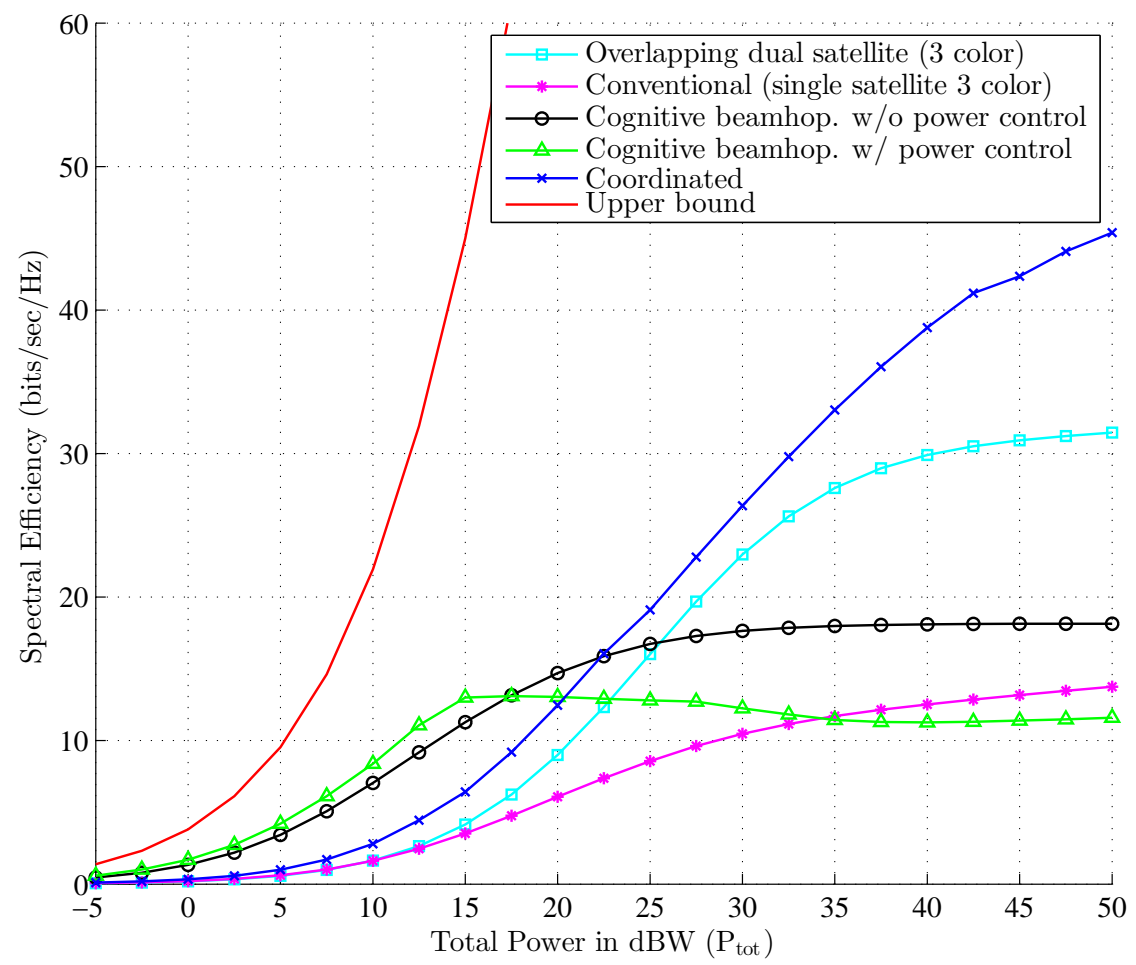

Figure 3: Spectral efficiency comparison of the considered schemes

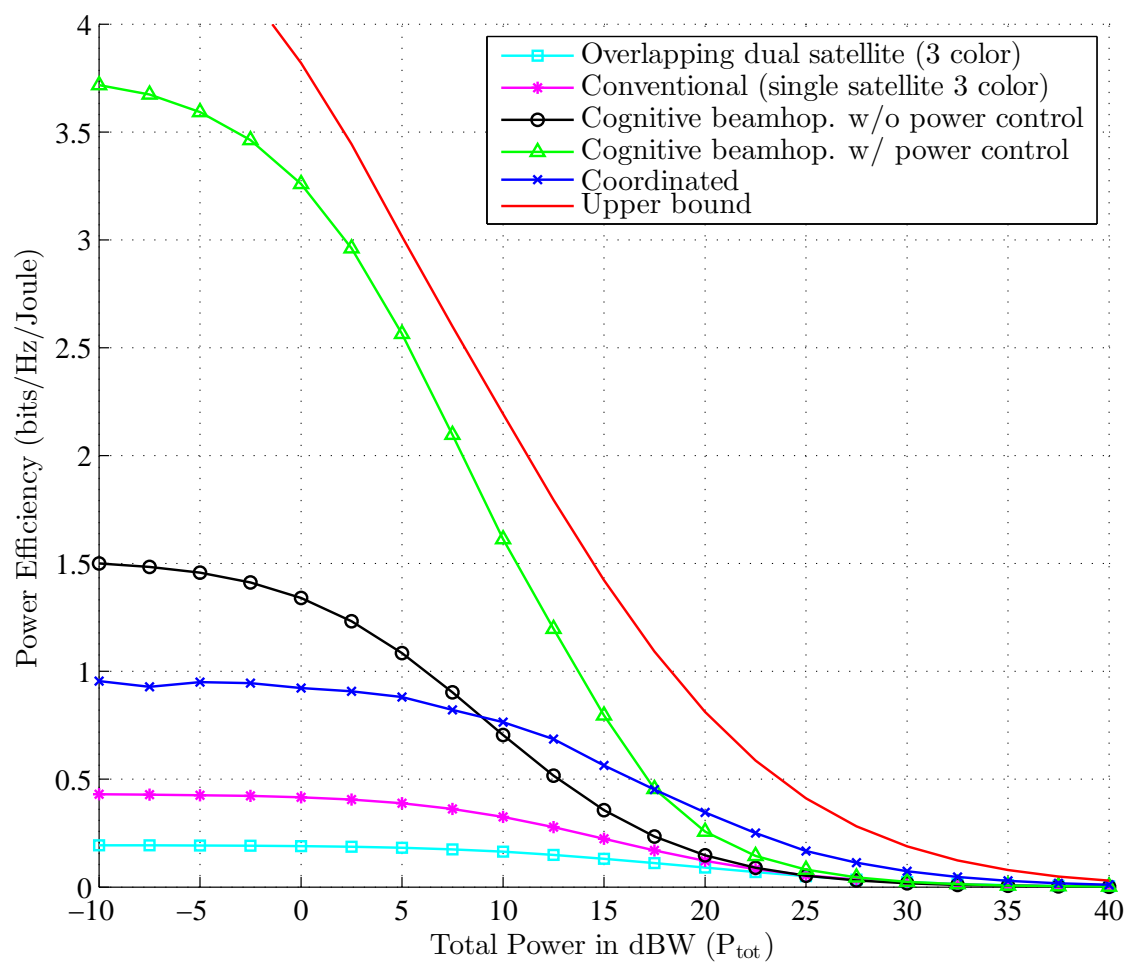

Figure 4: Power efficiency comparison of the considered schemes 


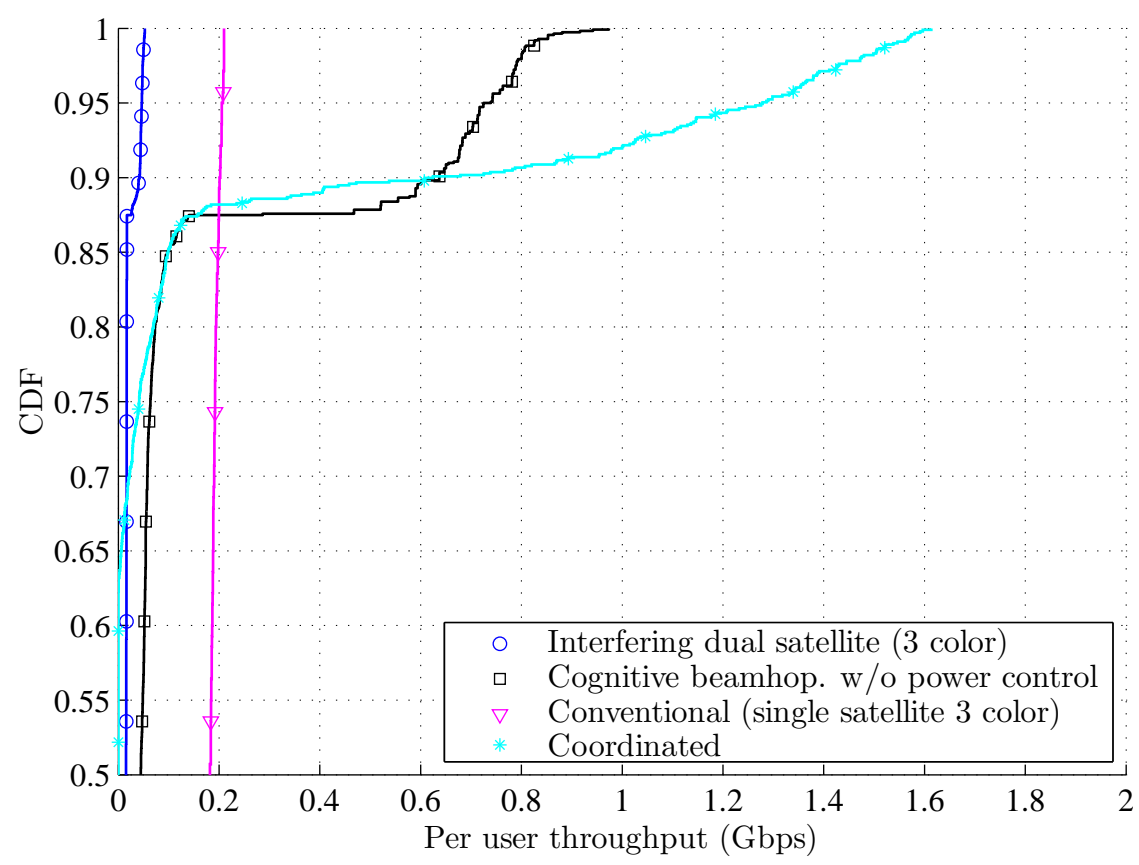

Figure 5: CDF of user throughput for the considered schemes $\left(P_{\text {tot }}=22.5 \mathrm{dBW}\right)$

In cooperative dual satellite system, full cooperation may require a high capacity backhauling link between the gateways in order to exchange both data and channel state information. In this context, techniques enabling partial cooperation should be applied in order to reduce the burden of the data exchange. The partial cooperation considered in this paper requires only the CSI information to be exchanged between the gateways instead of full data information. Unlike the terrestrial paradigm, the coordination in the satellite systems is easier since the satellite's ground operation is centralized at the gateway. Furthermore, the considered heuristic and iterative optimization algorithm requires full CSI information of all users. In practice, true CSI information may not be available and the uncertainty in CSI information needs to be taken into account. In the considered cognitive beamhopping system, the secondary satellite should be aware of the primary beamhopping pattern and timing information for synchronization purpose. Both coordianted and cognitive systems require a feedback link between their gateways in order to exchange this information. The main difference in the considered coordinated and cognitive approaches in terms of information exchange is that the information exchange between the gateways in the coordinated scheme is bidirectional whereas in the cognitive system, the information exchange is uni-directional i.e., the secondary system does not need to exchange any information to the primary. Another difference between these two schemes is that the coordinated scheme employs full frequency reuse whereas the cognitive system uses the slot reuse factor of $M$.

One might argue that one satellite may be redundant if both satellites belong to the same operator. However, in practice, two different satellites can be used for providing different services to same/different categories of users, hence enhancing the overall spectral efficiency of the system as mentioned in Section I. Further, there may arise a situation where the operator has to launch another satellite in order to meet the increased traffic demand in some coverage area. Another issue which may arise when two satellites belong to two different operators is that one operator may not be willing to share the CSI and data information in case of cooperative system and beamhopping sequence in the case of cognitive beamhopping system. In this context, a suitable strategy can be adopted depending on the employed spectrum assignment policy [27]. 


\section{VI.C. Conclusion and Future Works}

This paper has studied two novel satellite architectures, namely cooperative and cognitive, in order to enhance the spectral efficiency of the future SatCom systems. The performances of these schemes have been compared with those of the conventional multibeam and overlapping dual satellite systems in terms of the spectral efficiency, power efficiency and the user fairness. It can be concluded that the cognitive beamhopping based dual satellite system performs better in the low Signal to Noise Ratio (SNR) regime whereas the cooperative dual satellite system performs better in higher SNR regime. In terms of the power efficiency, both cognitive and cooperative schemes are more power efficient than the conventional multibeam and overlapping dual satellite systems. Further, cognitive beamhopping is found to be more power efficient than all other realizable schemes at the lower values of available total power and the cooperative scheme is found to be the best at higher values of the total available power. If the user fairness is the goal in designing the system, the conventional (single satellite) system is the best but can not support large number of users simultaneously as in dual satellite systems. Further, the second best approach in terms of fairness is overlapping dual satellite system but is the worst in terms of power efficiency. Moreover, cognitive beamhopping approach is more fair than the cooperative scheme whereas the cooperative scheme can provide higher rates to certain user groups.

In the considered user scheduling scheme for the cooperative dual satellite system, finding a set of users which can be simultaneously served by both the satellites while fulfilling minimum rate requirements is an important research challenge. In the context of cognitive dual satellite system, finding a proper switching sequence for the secondary system in order to increase its throughput while respecting the interference threshold of the primary users is an important future aspect. Further, the combination of dynamic spectrum sensing as well as the EZ principle for exploiting spatio-temporal spectral holes can be considered as another important future research direction.

\section{Acknowledgement}

This work was supported by the National Research Fund, Luxembourg under the CORE projects "CO${ }^{2} \mathrm{SAT}$ : Cooperative and Cognitive Architectures for Satellite Networks" and "SeMIGod: SpEctrum Management and Interference mitiGation in cOgnitive raDio satellite networks".

\section{References}

${ }^{1}$ P. Chini, G. Giambene, and S. Kota, "A survey on mobile satellite systems," Int. J. Satell. Commun. Network, vol. 28, no. 1, pp. 29-57, 2009.

${ }^{2}$ O. Vidal, and et al, "Next generation High Throughput Satellite system," in Proc. IEEE First AESS European Conf. on Satellite Telecommun. (ESTEL), pp. 1-7, Oct. 2012.

${ }^{3}$ G. Zheng, S. Chatzinotas, and B. Ottersten, "Generic Optimization of Linear Precoding in Multibeam Satellite Systems," IEEE Trans. Wireless Commun., vol. 11, no. 6, pp. 2308-2320, June 2012.

${ }^{4}$ D. Christopoulos, S. Chatzinotas, G. Zheng, J. Grotz, and B. Ottersten, "Linear and nonlinear techniques for multibeam joint processing in satellite communications", EURASIP J. Wireless Commun. Network, vol. $162,2012$.

${ }^{5}$ S. K. Sharma, S. Chatzinotas, and B. Ottersten, "Satellite cognitive communications: Interference modeling and techniques selection," in Proc. 6th ASMS and 12th SPSC, pp. 111-118.

${ }^{6}$ D. Christopoulos, S. Chatzinotas, and B. Ottersten, "User scheduling for coordinated dual satellite systems with linear precoding," in Proc. IEEE ICC, 2013, pp. 4498-4503.

${ }^{7}$ S. K. Sharma, S. Chatzinotas, and B. Ottersten, "Cognitive beamhopping for spectral coexistence of multibeam satellites," in Proc. Int. Conf. FUNMS, July 2013, pp. 1-10.

${ }^{8}$ R. Jain, D. Chiu, and W. Hawe, "A Quantitative Measure of Fairness and Discrimination for Resource Allocation in Shared Systems", Digital Equipment Corporation, Technical Report DEC-TR-301, Tech. Rep., 1984.

${ }^{9}$ M. Diaz, N. Courville, C. Mosquera, G. Liva, and G. Corazza, "Nonlinear interference mitigation for broadband multimedia satellite systems," in Int. Workshop on Satellite and Space Comm., Sept. 2007, pp. 61-65.

${ }^{10}$ S. K. Sharma, S. Chatzinotas, and B. Ottersten, "Spectrum sensing in dual polarized fading channels for cognitive SatComs," in IEEE GLOBECOM, 2012, pp. 3419-3424.

${ }^{11}$ S. Kandeepan, L. De Nardis, M.-G. Di Benedetto, A. Guidotti, and G. Corazza, "Cognitive satellite terrestrial radios," in Proc. IEEE GLOBECOM, Dec. 2010, pp. 1-6. 
${ }^{12}$ S. K. Sharma, S. Chatzinotas, and B. Ottersten, "Spatial filtering for underlay cognitive SatComs," in Proc. 5th Int. Conf. PSATS, June 2013.

${ }^{13}$ S. K. Sharma, S. Chatzinotas, and B. Ottersten, "Transmit beamforming for spectral coexistence of satellite and terrestrial networks" in Proc. 8th Int. Conf. CROWNCOM, July 2013, pp. 275-281.

${ }^{14}$ Y. H. Yun, and J. H. Cho, "An orthogonal cognitive radio for a satellite communication link", in Proc. IEEE 20th Int. Symp. PIMRC, Sept. 2009, pp. 3154-3158.

${ }^{15}$ S. K. Sharma, S. Chatzinotas, and B. Ottersten, "Exploiting polarization for spectrum sensing in cognitive SatComs," in Proc. 7th Int. Conf. CROWNCOM, 2012, pp. 36-41.

${ }^{16} \mathrm{D}$. S. Oh, and et al "A study on the separation distance for frequency sharing between GSO network and terrestrial network in Ka band" in Proc. IEEE VTC, Singapore, May 2008, pp. 2967-2971.

${ }^{17}$ S. K. Sharma, S. Chatzinotas, and B. Ottersten, "Interference alignment for spectral coexistence of heterogeneous networks," EURASIP J. Wireless Commun. and Networking, vol. 46, 2013.

${ }^{18}$ L. N. Wang, B. Wang, "Distributed power control for cognitive satellite networks" Advanced Materials Research: Mechatronics and Intelligent Materials II, March 2012, vol. 71, pp. 1156-1160.

${ }^{19}$ S. Chatzinotas, S. K. Sharma, and B. Ottersten, "Frequency packing for interference alignment-based cognitive dual satellite systems," to appear in proc. VTC Fall-2013, Sept. 2013.

${ }^{20}$ S. K. Sharma, S. Chatzinotas, and B. Ottersten, "Inline Interference Mitigation Techniques for Spectral Coexistence of GEO and NGEO Satellites", in Proc. AIAA ICSSC 2013, Florence, Italy, Oct. 2013.

${ }^{21}$ D. Tarchi, and et al, "Technical Challenges for Cognitive Radio Application in Satellite Communications," in Proc. Int. Conf. CROWNCOM, Oulu, Finland, June 2014.

${ }^{22} \mathrm{~J}$. Anzalchi, and et al "Beam hopping in multi-beam broadband satellite systems: System simulation and performance comparison with non-hopped systems," in Proc. 5th ASMS and 11th SPSC, Sept. 2010, pp. 248-255.

${ }^{23}$ R. Alegre-Godoy, N. Alagha, and M. Vazquez-Castro, "Offered capacity optimization mechanisms for multibeam satellite systems," in Proc. IEEE Int. Conf. Commun., June 2012, pp. 3180-3184.

${ }^{24} \mathrm{~J}$. Anzalchi J, and et al, "Beam hopping in multi-beam broadband satellite systems: System simulation and performance comparison with non-hopped systems" in Proc. 5th ASMS conf. and the 11th SPSC workshop, Cagliari, Italy, Sept. 2010, pp. $248-255$.

${ }^{25}$ N. Fonseca, and J. Sombrin, "Multi-beam reflector antenna system combining beam hopping and size reduction of effectively used spots" IEEE Antennas and Propagation Magazine, vol. 54, no. 2, pp. 88-99, April 2012.

${ }^{26}$ L. Jiang, "Multi-beam satellite resource optimization for beam hopping transmission" PhD thesis, Department of Telecommun. and systems Engineering, Universitat Autonoma de Barselona, 2010.

${ }^{27}$ S. K. Sharma, S. Chatzinotas, and B. Ottersten, "Cognitive beamhopping for spectral coexistence of multibeam satellites," in Int. J. Satell. Commun. Network, March 2014.

${ }^{28}$ M. Schneider, C. Hartwanger, and H. Wolf, "Antennas for multiple spot beam satellites" CEAS Space Journal, vol. 2, pp. 59-66, Dec. 2011.

${ }^{29}$ S. Huaizhou, and et al, "Fairness in Wireless Networks: Issues, Measures and Challenges," IEEE Communications Surveys and Tutorials, vol. 16, no. 1, pp. 5-24, 2014.

14 of 14 